\title{
Lohnt es sich, die Hypothek zu amortisieren?
}

R. Bütler

Korrespondenz:

Roland Bütler, dipl. Treuhandexperte FMH Services Treuhand Geschäftsstelle Muri AG Kirchenfeldstrasse 6

CH-5630 Muri

Tel. 0566640309

Fax 0566645566

E-Mail:

roland.buetler@fmhtreuhand.ch
Der Kauf eines Einfamilienhauses oder eines Stockwerkeigentums erfolgt fast immer unter Zuhilfenahme von Fremdkapital. Dieses wird in Form von Hypotheken über Bank- bzw. Versicherungsinstitute aufgenommen. In der Regel muss ein Teil der Hypotheken zurückbezahlt werden. Diese Amortisation kann grundsätzlich auf zwei Arten geschehen: direkt oder indirekt. In der Regel finanzieren die Hypothekargläubiger Eigenheime mit einem Fremdkapital bis maximal 80\% des Verkehrswertes. Etwa 65\% des Verkehrswertes wird über eine 1. Hypothek ohne Verpflichtung zur Amortisation gewährt. Die Differenz zwischen der 1. Hypothek und der Gesamtbelehnung wird als 2. Hypothek bezeichnet, welche in der Regel über einen bestimmten Zeitraum amortisiert werden muss.

Viele Eigenheimbesitzer amortisieren ihre 2. Hypothek indirekt, d.h., sie bezahlen die Amortisation nicht dem Hypothekargläubiger, sondern sie bedienen sich der Säule 3a bzw. teilweise der Säule 3b, seltener der Säule 2 (mittels Verpfändung der Vorsorgegelder). Eine indirekte Amortisation mittels der Säule 3a oder Säule 3b lohnt sich zum heutigen Zeitpunkt immer, da einerseits der einbezahlte Betrag steuerlich abziehbar und das Kapital steuerlich privilegiert ist und andererseits auch Vorsorgeleistungen damit abgedeckt werden.

Seit einigen Jahren haben sich die Rahmenbedingungen an den Finanzmärkten (Zinsniveau) grundlegend geändert. Deshalb drängt sich die Frage auf, ob sich eine freiwillige Amortisation überhaupt lohnt.

Private Schuldzinsen sind nach geltendem Recht (vgl. Stabilisierungsprogramm 98) vom Einkommen steuerlich absetzbar. Viele Eigen- heimbesitzer amortisieren ihre 2. Hypothek mittels indirekter Amortisation. Weitere Amortisationen werden in der Regel nicht getätigt, weil ein Grossteil der Eigenheimbesitzer nach wie vor glaubt, dass man Schulden haben muss, um eine Steuerersparnis zu erreichen. Das folgende Beispiel dient zur Verdeutlichung, dass dies nicht immer stimmen muss:

1. Hypothek à 3,00\% bzw. Zins Fr. 60000018000

2. Hypothek à 3,50\% bzw. Zins Fr. $200000 \quad 7000$

Fremdkapital insgesamt bzw. Zins Fr. 80000025000

Da die Schuldzinsen von insgesamt Fr. 25 000.vom steuerbaren Einkommen absetzbar sind, reduziert sich die Steuerbelastung bei einem Grenzsteuersatz von etwa 30\% um Fr. 7500.- pro Jahr, somit auf Fr. 17500.-. Der durchschnittliche Zinssatz der Hypotheken beträgt 3,125\%. Unter der Voraussetzung, dass der Hypothekarschuldner noch freie Eigenmittel hat und eine jährliche Rendite nach Steuern von mehr als $2,188 \%$ erzielt (notabene risikolos), macht es meiner Meinung nach Sinn, die Verschuldung konstant zu halten und das Kapital anzulegen. Beim heutigen Zinsniveau und den volatilen Finanzmärkten dürfte es jedoch nicht ohne Risiko gehen, um eine Rendite von über $2,1 \%$ zu realisieren.

Zusammenfassend kann festgehalten werden, dass sich eine freiwillige Amortisation dann lohnt, wenn aus dem verfügbaren Eigenkapital eine geringere Nettorendite nach Steuern erzielt wird, als Sie für das Fremdkapital an Schuldzinsen nach Steuern bezahlen. 